\title{
Quantitative Measurement of Immunoglobulins and Free Light Chains Using Mass Spectrometry
}

\author{
Martijn M. VanDuijn, ${ }^{\dagger, \|}$ Joannes F. M. Jacobs, ${ }^{\ddagger, \|}$ Ron A. Wevers, ${ }^{\S}$ Udo F. Engelke, ${ }^{\S}$ Irma Joosten, $^{\ddagger}$ \\ and Theo M. Luider ${ }^{*}$, \\ ${ }^{\dagger}$ Laboratory of Neuro-Oncology and Clinical \& Cancer Proteomics, Department of Neurology, Erasmus MC, Wytemaweg 80, \\ 3015CN Rotterdam, The Netherlands \\ ${ }^{\ddagger}$ Laboratory of Medical Immunology, Department of Laboratory Medicine, Radboudumc, Nijmegen, The Netherlands

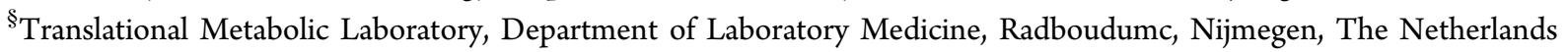

\section{Supporting Information}

ABSTRACT: Serum free light chain (sFLC) assays are well established in the diagnosis and monitoring of plasma cell disorders. However, current FLC immunoassays are subject to several analytical issues, which results in a lack of harmonized results. To facilitate sFLC standardization, we investigated the strengths and limitations of mass spectrometry as a novel technological platform for sFLC quantification. Stable isotope labeled reference peptides are added to serum samples for quantitation by selected reaction monitoring (SRM). The use of redundant peptide sets allows for quality control measures during data analysis. Measurements on serum provide information on intact immunoglobulins, but depletion of these intact molecules from the sera during sample processing permits the quantitation of sFLC. sFLC concentrations measured with SRM were comparable to those obtained by nephelometry and showed excellent linearity $\left(r^{2}>0.99\right)$. In samples with high levels of sFLC, SRM data was more consistent with serum protein electrophoresis than nephelometric data and SRM is unaffected by antigen excess. The lower limits of quantitation were 3.8 and $2.7 \mathrm{mg} / \mathrm{L}$ for $\kappa$ and $\lambda$ sFLC. Errors due to polymorphic sequences were prevented by comparison of redundant peptide pairs. The application of stable isotope labeling combined with SRM can overcome many of the current potential analytical issues of sFLC analysis. We describe which hurdles still need to be taken to make SRM a robust and more accurate method for sFLC measurements.

$\mathrm{M}$ onoclonal gammopathies are characterized by the clonal expansion of plasma cells in the bone marrow and by the production of monoclonal immunoglobulins (M-proteins). These disorders range from benign, monoclonal gammopathy of undetermined significance, to symptomatic myeloma with bone destruction, bone marrow suppression, and renal damage. The International Myeloma Working Group has established criteria for the diagnosis of these plasma cell dyscrasias. ${ }^{1,2}$ The detection and quantification of M-proteins involves serum and urine electrophoresis and immunofixation, nephelometric analysis of serum immunoglobulins and of serum free light chain (sFLC). The quantification of M-proteins and monoclonal sFLC is technically challenging, which is among others demonstrated by considerable intermethod differences. ${ }^{3-8}$ Accurate and sensitive quantification is essential for the monitoring of monoclonal gammopathies. ${ }^{9}$ Here, we present a quantitative $\mathrm{LC}-\mathrm{MS}$ procedure that allows us to characterize both intact immunoglobulins and their free light chains in serum.

Free Light Chains. Plasma cells synthesize approximately $40 \%$ excess of light chains to allow efficient assembly of the immunoglobulin heavy and light chain heterodimer. The excess of light chains is excreted into the circulation as sFLC and finally cleared renally. ${ }^{10}$ In patients with impaired renal clearance, sFLC may also be elevated in absence of a plasma cell dyscrasia, albeit not in a monoclonal fashion. The clonal character is reflected in the ratio between the $\kappa$ and $\lambda$ chains of the sFLC. ${ }^{11}$

Existing Tests. Measurement of sFLC has become well established in the diagnosis and follow-up of monoclonal gammopathies improving the screening, the risk-assessment, and the evaluation of response to therapy. ${ }^{9,12,13}$ sFLC analysis in the clinics is dominated by nephelometric and turbidometric immunoassays. ${ }^{11,12}$ However, quantification of sFLC by these methods is subject to difficulties such as inconsistency in linear responses, imprecision caused by lot-to-lot variation and interanalyzer variation, poor accuracy, and antigen-excess. $^{4-6,14-18}$ Recently a second commercial FLC assay was introduced which is based on monoclonal detection reagents. ${ }^{19}$ Because of significant differences in sFLC concentrations between nephelometric tests from both vendors, they cannot be used interchangeably in individual patients. ${ }^{19-22}$ Measurement of sFLC and its standardization is difficult because each

Received: April 3, 2015

Accepted: July 13, 2015

Published: July 13, 2015 
individual patient has a unique monoclonal FLC, with considerable heterogeneity in size, charge, isoelectric point, and concentration of FLCs. ${ }^{4,23}$ Lack of harmonization is further magnified by the diversity of polyclonal and monoclonal-based immunochemical FLC-methods. An internationally accepted FLC reference-method or -material is currently not available. Since diagnosis, prognosis, and monitoring of patients rely on accurate sFLC measurements, standardization is urgently needed.

The LC-MS method described in this paper quantifies sFLC in a fundamentally different manner than nephelometric immunoassays, as it relies on the measurement of the concentration of proteolytic peptides from the analyte, rather than an affinity interaction of a nephelometry reagent with an epitope on the analyte. Thus, our method may not share the problems of nephelometry described above. The role for mass spectrometry in immunoglobulin quantification was recently expanded by several groups who describe an LC-MS method to quantify IgG subclasses using SRM. ${ }^{24-27}$ While similar to the current method with regard to intact immunoglobulins, our capability to quantify sFLC is an important addition. Moreover, the method in this paper relies on an extended set of peptides, which increases robustness and the possibilities for quality control.

\section{EXPERIMENTAL SECTION}

Chemicals were obtained from Sigma-Aldrich (St. Louis, MO) unless reported otherwise.

Reference Standards. For quantification, stable isotope labeled peptides were obtained as internal standards for peptides for the immunoglobulin chains of interest (Pepscan BV, Lelystad, The Netherlands). ${ }^{28}$ Peptide amount and purity were established by amino acid analysis, HPLC, and MS by the manufacturer. In addition, some peptides were analyzed by quantitative NMR (qNMR) using a $500 \mathrm{MHz}$ instrument (Bruker, The Woodlands, TX), maleic acid as qNMR reference, trimethylsilyl propanoic acid as chemical shift reference, and $\mathrm{D}_{2} \mathrm{O}$ as a lock signal.

Certified Reference Material (CRM) ERM-DA470k/IFCC was included as a serum protein reference (Institute for Reference Materials and Measurements, Belgium). ${ }^{29}$ The sensitivity of the SRM method was assessed by titrating purified human $\kappa$ and $\lambda$ free light chains (Bethyl Laboratories, Montgomery, TX) in a serum matrix from an aplastic phase following stem cell transplantation, low in endogenous immunoglobulins.

Patient Cohort. Sera were derived from routine diagnostic samples of which sFLC analysis was requested at the Department of Laboratory Medicine at the Radboudumc, Nijmegen, The Netherlands. Aliquots were archived at $-80{ }^{\circ} \mathrm{C}$ after collection and thawed directly before further analysis. Sera were retrospectively selected to obtain a cohort of samples with a wide range of both $\kappa$ FLC and $\lambda$ FLC, with or without the presence of intact monoclonal immunoglobulins. Sera were derived from healthy controls, patients with chronic kidney disease, and patients with a variety of monoclonal gammopathies. All samples and clinical data were coded and anonymized as specified in the Dutch code of conduct for biomedical research.

Conventional M Protein Analysis. For M-protein quantification and characterization, agarose gel electrophoresis and immunofixation electrophoresis were performed on the Hydrasys (Sebia, Evry, France) using reagents from Sebia according to the manufacturer's protocol. Nephelometric serum IgG, IgA, and IgM were measured on an Immage 800 analyzer (Beckman Coulter, Fullerton, CA) according to the manufacturers' protocol. Both Freelite FLC (The Binding Site, Birmingham, U.K.) and N Latex FLC (Siemens, Marburg, Germany) assays were performed on a BNII analyzer according to the manufacturer's protocols. The reference ranges for both assays were as published previously. ${ }^{19,30}$

Sample Preparation. Sera were cleared for $5 \mathrm{~min}$ at $20000 \mathrm{~g}$ in a centrifuge and, as serum volumes were limited, a small $4 \mu \mathrm{L}$ aliquot was taken. The serum was diluted in 1200 $\mu \mathrm{L}$ of PBS containing reference peptides at $16.6 \mathrm{nM}$ each. For the quantitation of intact immunoglobulins, this material was used directly for reduction, alkylation, and digestion; for the quantitation of sFLC, intact immunoglobulins were first depleted from the sample.

To deplete intact immunoglobulin G, agarose beads coupled to protein A and G (Thermo Fisher Scientific, MA) were used. To also deplete IgA, goat antihuman IgA (Jackson Immunoresearch Europe, U.K.) was included, immobilized on the protein A/G beads before addition of serum samples. Additional depleting antibodies could be included in a similar manner if required. We prepared and measured samples in duplicate for the depleted samples and included one sample with undepleted serum for each patient. The diluted sera were incubated for $1 \mathrm{~h}$ with the Protein A/G beads $(15 \mu \mathrm{L}$ packed beads $/ 75 \mu \mathrm{L}$ diluted serum), after which a filtrate was collected. A volume of $30 \mu \mathrm{L}$ was diluted in $30 \mu \mathrm{L}$ of $50 \mathrm{mM}$ ammonium bicarbonate, $10 \mathrm{mM}$ DTT, $0.2 \%$ Rapigest SF (Waters, MA). After reduction at $60{ }^{\circ} \mathrm{C}$ for $30 \mathrm{~min}$, cysteine residues were alkylated with $15 \mathrm{mM}$ iodoacetamide $\left(15 \mathrm{~min}, 25^{\circ} \mathrm{C}\right)$. Finally, $30 \mathrm{mM}$ DTT and $600 \mathrm{ng}$ of trypsin (Promega, WI) were added, quenching the alkylation and digesting overnight at $37{ }^{\circ} \mathrm{C}$. Trifluoroacetic acid was added to $0.5 \%$, after centrifugation ( 5 min $20000 \mathrm{~g}$ ) samples were injected on the LC-MS. The procedure has been summarized in Figure 1.

LC-MS. Chromatography was performed on a nano-LC (Dionex Ultimate 3000, ThermoFisher Scientific, MA). Samples were separated on a C18 column (Dionex Acclaim PepMap100, $3 \mu \mathrm{m}, 100 \AA$, $75 \mu \mathrm{m}$ i.d. $\times 15 \mathrm{~cm}$ ) using a gradient of $0.1 \%$ formic acid to $80 \%$ acetonitrile with $0.1 \%$ formic acid.

Mass spectrometry was performed on a Qtrap 4000 mass spectrometer (AB Sciex, MA) with a nanospray source. Table S-1 in the Supporting Information shows all SRM peptides and the associated masses used in the method, typically three transitions per peptide. We used a scheduled SRM method with a $200 \mathrm{~s}$ measurement window around the predicted retention time, as defined with a test run of the peptide standards and the iRT algorithm in the Skyline software package. The Analyst mass spectrometer software determines the dwell times based on a $3 \mathrm{~s}$ cycle time. Additional measurements were performed on a Xevo TQ-S (Waters, MA) to compare portability and sensitivity or on a LTQ Orbitrap XL (Thermo Fisher Scientific, MA) to identify polymorphisms.

Data Processing. Signals were integrated using Skyline and exported for further analysis. ${ }^{31}$ The concentration of each peptide was determined based on the signal ratio between the endogenous peptide and the spiked internal standard peptide. Subsequently, peptide concentrations were aggregated to the protein level by summing sequence variants and averaging peptides belonging to the same protein chain. This process has been illustrated by equations in Figure S-1 in the Supporting Information. No corrections were applied for intact immuno- 


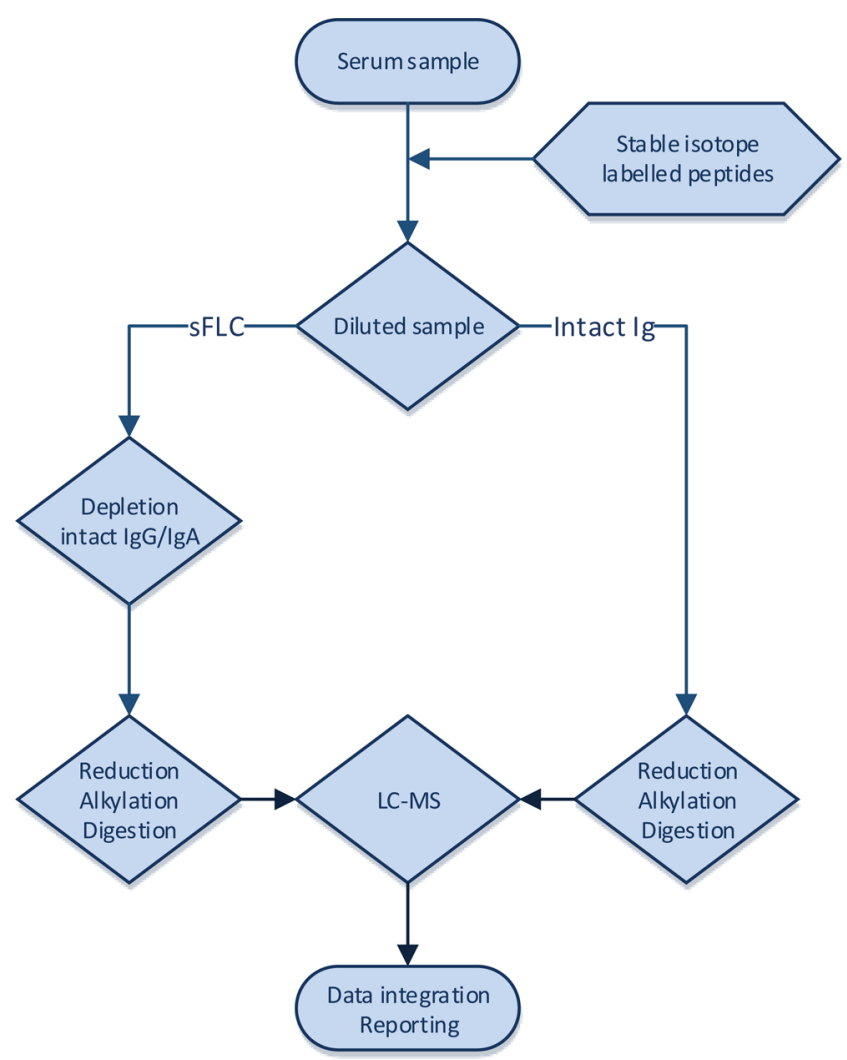

Figure 1. Flowchart of the sample preparation used to quantify immunoglobulins and sFLC using selected reaction monitoring.

globulins remaining after depletion, as the relative contribution of $\kappa$ and $\lambda$ light chains cannot be determined. De novo sequencing of orbitrap data was performed using Peaks Studio 6 (Bioinformatics Solutions, ON, Canada).

Quality Control. Several transitions are included to monitor potential chemical modifications and integrity of the selected peptides. As peptides with cysteine or methionine had to be included, controls for potential artifacts were measured. The oxidation of methionines is monitored by measuring an oxidized peptide, the degree of alkylation of cysteines by measuring an unalkylated peptide, and the degree of digestion by measuring several peptides containing a missed cleavage. A list of these peptides for quality control is incorporated in Table $\mathrm{S}-1$ in the Supporting Information, labeled as "QC" in the protein column. The results from these $\mathrm{QC}$ measurements was monitored for outliers or unusual patterns. Furthermore, the reliability of the data is assessed by comparing results for peptides belonging to the same protein. Inconsistencies between such cognate sets indicate analytical artifacts or sequence polymorphisms. Within a peptide, the ratio of the contributing fragment ions should remain similar between samples. The success of the depletion of intact immunoglobulins has been assessed through peptides from the heavy chain.

Safety Considerations. Serum samples are a biohazardous material and should be treated as such.

\section{RESULTS AND DISCUSSION}

Serum Depletion. Immunoglobulin light chains exist in serum unbound as part of intact immunoglobulins or as free light chains. After proteins are digested with a protease, it is no longer possible to distinguish light chain peptides derived from intact immunoglobulins from those that existed as free light chains. As sFLC are typically present in a much lower concentration than total immunoglobulins $(10 \mathrm{mg} / \mathrm{L}$ sFLC vs $10 \mathrm{~g} / \mathrm{L}$ intact immunoglobulins for healthy individuals), the contribution of free light chains cannot be determined reliably from the stoichiometry between heavy and light chains. Also, it is important to measure the ratio between $\kappa$ and $\lambda$ sFLC, while results would reflect a ratio from sFLC and intact immunoglobulins combined. It is therefore necessary to deplete the intact immunoglobulins from the sample. Undepleted samples were still measured, as they remain valuable to help the interpretation of sFLC data and for additional diagnostic capabilities for abnormalities in intact immunoglobulins and rare heavy chain gammopathies. ${ }^{2432}$ The depletion was effective for both IgG and IgA, leaving on average $0.75 \%$ of the initial amount for IgG (Figure S-2 in the Supporting Information). The amount of IgG remaining after depletion still results in a detectable signal of associated light chains which, while mostly below the level of (free) light chains in healthy subjects, is not desirable and may be improved upon.

Sensitivity, Linearity, and Variation. The sensitivity of the assay is determined by the combination of measurements made for the peptides in the SRM set. The result of several peptides is averaged in the result of a protein chain, and such aggregated data was used to assess the lower limit of quantitation (LLoQ) of the assay.

We tested the sensitivity by spiking purified $\kappa$ and $\lambda$ light chains in a serum matrix low in endogenous immunoglobulins. The LLoQ was defined as $10 \times \mathrm{SD}_{\text {blank }} /$ slope and was $3.8 \mathrm{mg} / \mathrm{L}$ for $\kappa$ and $2.7 \mathrm{mg} / \mathrm{L}$ for $\lambda$ chains. As can be seen from Figure 2,

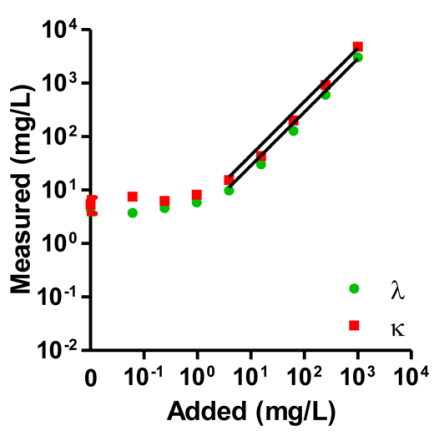

Figure 2. LLoQ of the sFLC assay. Purified sFLC of both $\kappa$ and $\lambda$ type were titrated in a matrix of an aplastic serum sample and the concentration of sFLC was determined by SRM. The linear curve fit is based on spiked concentrations of sFLC of $4 \mathrm{mg} / \mathrm{L}$ and higher.

the results were linear over 3 logs $\left(r_{\lambda}^{2}=0.996 ; r_{\kappa}^{2}=0.993\right)$ until signals were lost. For the best performing peptides, a small signal could be observed in the aplastic serum alone, suggesting endogenous sFLC levels in this matrix on the order of $1 \mathrm{mg} / \mathrm{L}$ for both $\kappa$ and $\lambda$ chains. Reanalysis of the samples on a different mass spectrometer (Xevo TQ-S, Waters, MA, data not shown) resulted in data with a LLoQ of $0.7 \mathrm{mg} / \mathrm{L}$ for $\kappa$ and $0.9 \mathrm{mg} / \mathrm{L}$ for $\lambda$ and indicated an endogenous sFLC level of $2 \mathrm{mg} / \mathrm{L}$ in the aplastic serum.

On the basis of the samples spiked with more than the LLoQ, the average CV was $6.6 \%$ for $\kappa$ and $2.9 \%$ for $\lambda$. However, CVs were higher for the replicates that were measured in the cohort, on average $19.7 \%$ and $13.6 \%$ for $\kappa$ and $\lambda$, respectively. This may be related to the low median sFLC concentration in the cohort ( 15 and $34 \mathrm{mg} / \mathrm{L})$, to variations associated with the handling of a larger number of samples or to the longer time 
lapsed between injection of the replicated on the LC-MS system.

Cross-Checks and Quality Controls. Results from most peptides can be compared to their cognate counterparts from the same protein chain for consistency. We compared several such peptide sets, as illustrated in Figure 3. For a given protein

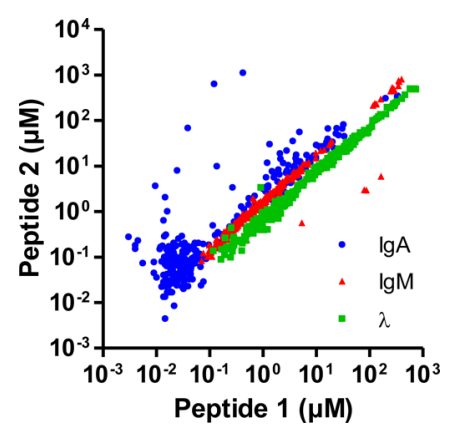

Figure 3. Correlation of cognate peptide pairs. The concentration of peptide pairs derived from the same protein chain for sera from the cohort and pairs from IgA (SAVQGPPER and YLTWASR), IgM (VTSTLTIK and YVTSAPMPEPQAPGR), and the $\lambda$ light chain (YAASSYLSLTPEQWK and SYSCQVTHEGSTVEK) are shown.

concentration, the same concentration is expected for both peptides derived from it. While most peptides showed a good correlation with each other, some data points deviate from the general distribution. This may indicate sequence polymorphisms in that patient, as discussed in the following section. In addition, the measurements do not align perfectly to the diagonal that represents identical outcomes, indicating that some inaccuracies remain in the calibration of some peptides. Where possible, an additional characterization was performed on the peptide standards by qNMR. Most measurements agreed with the manufacturer's data, but deviations up to $40 \%$ were also observed. The manufacturer's data was used as the basis for all quantitation, and the qNMR results were included in Table $S-1$ in the Supporting Information.

Signals from the oxidized form of the peptide DTLMISR amounted to $1-2 \%$ of that of the normal form and were stable over time (Figure S-3A in the Supporting Information). We do not have calibrated data for the oxidized form, but assuming that the detector response is similar for both peptides, the ratio found was deemed acceptable. Moreover, the ratio did not change much during the measurement period (22 days), and any oxidation events would equally affect endogenous and standard peptides. Thus, methionine oxidation was not a problem in this data set. From the same measurements, we did see a gradual decrease in signal from both the endogenous peptide and the standard peptide. This suggests that the peptide is slowly lost from the sample by another mechanism, for example, adsorption to the vial wall. Again, this is corrected for by the internal standard, but nevertheless it is recommended to measure samples within days or at most weeks after preparation is complete. We also compared a tryptic peptide with an extended peptide with a missed cleavage. The miscleaved peptide signal was correlated $\left(R^{2} 0.91\right)$ to the tryptic peptide, suggesting that a constant fraction of the protein evades digestion (Figure S-3B in the Supporting Information). As the relative amount of these unwanted forms of the peptides was low we conclude that neither these artifacts, nor the missed alkylation of cysteines residues (Figure S-3C in the Supporting Information), had a significant adverse effect on the data.

Polymorphisms. Peptides were chosen to cover the sequence variants found in humans as reported in the IMGT database. ${ }^{33}$ However, rare or novel sequence variants may still occur, and the occurrence of a polymorphic peptide will affect the quantitation in the SRM assay. In order to account for polymorphisms, two or more peptides per protein chain are quantified, which will reveal a polymorphism as a discrepancy in the data. To further characterize polymorphisms, we analyzed a subset of 37 samples on an Orbitrap mass spectrometer in order to elucidate suspected variants of immunoglobulin peptides. The data set was analyzed using a protein database consisting of known SNPs from the hg19 reference genome as well as by de novo sequencing of the MS/MS data. ${ }^{34,35}$ The most frequent polymorphism affecting our data occurred in the IgA peptide SAVQGPPER to SAVQGPPDR. This variant was absent in the IMGT database but can be found in databases such as Kabat. ${ }^{36}$ Reanalysis of the original LC-MS samples with transitions for the mutated peptide showed evidence for the variant peptide in $39 \%$ of the samples (34\% reported in the ExAC database). In addition, we found in one serum a possible polymorphism for IgM, YVTSAPMPEPQAPGR to YVTSAPVPEPQAPGR. Finally, the ExAC exome database was screened for additional polymorphisms in peptides of interest. ${ }^{37}$ Polymorphisms with a prevalence of more than $1 \%$ that were
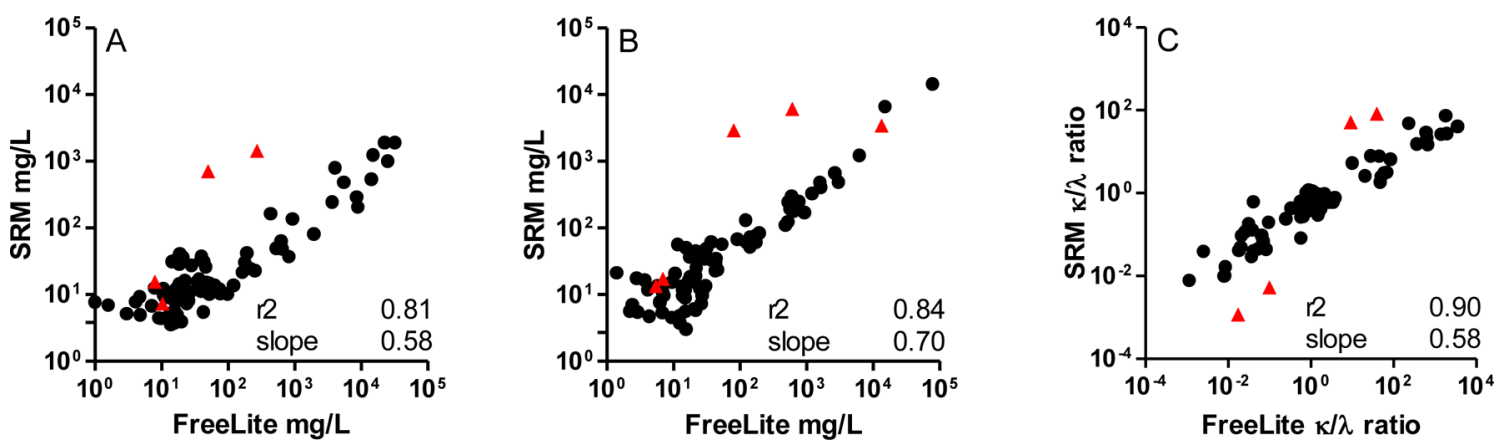

Figure 4. Comparison between the SRM test for sFLC and the commercial Freelite assay for $\kappa$ sFLC (A), $\lambda$ sFLC (B), and $\kappa / \lambda$ ratio (C). Shown are the results for those samples from the cohort that have data available from both tests in the comparison. Data points in red triangles contain intact immunoglobulin classes that could not be depleted with the current protocol and add signal to our light chain quantitation. Figure S-4 in the Supporting Information shows similar comparisons between SRM, Freelite, and N-Latex data. Each plot shows the slope and $r^{2}$ after linear regression on log-transformed data (black markers only). The LLoQ of the SRM method is shown by an additional tick on the $Y$-axis. 

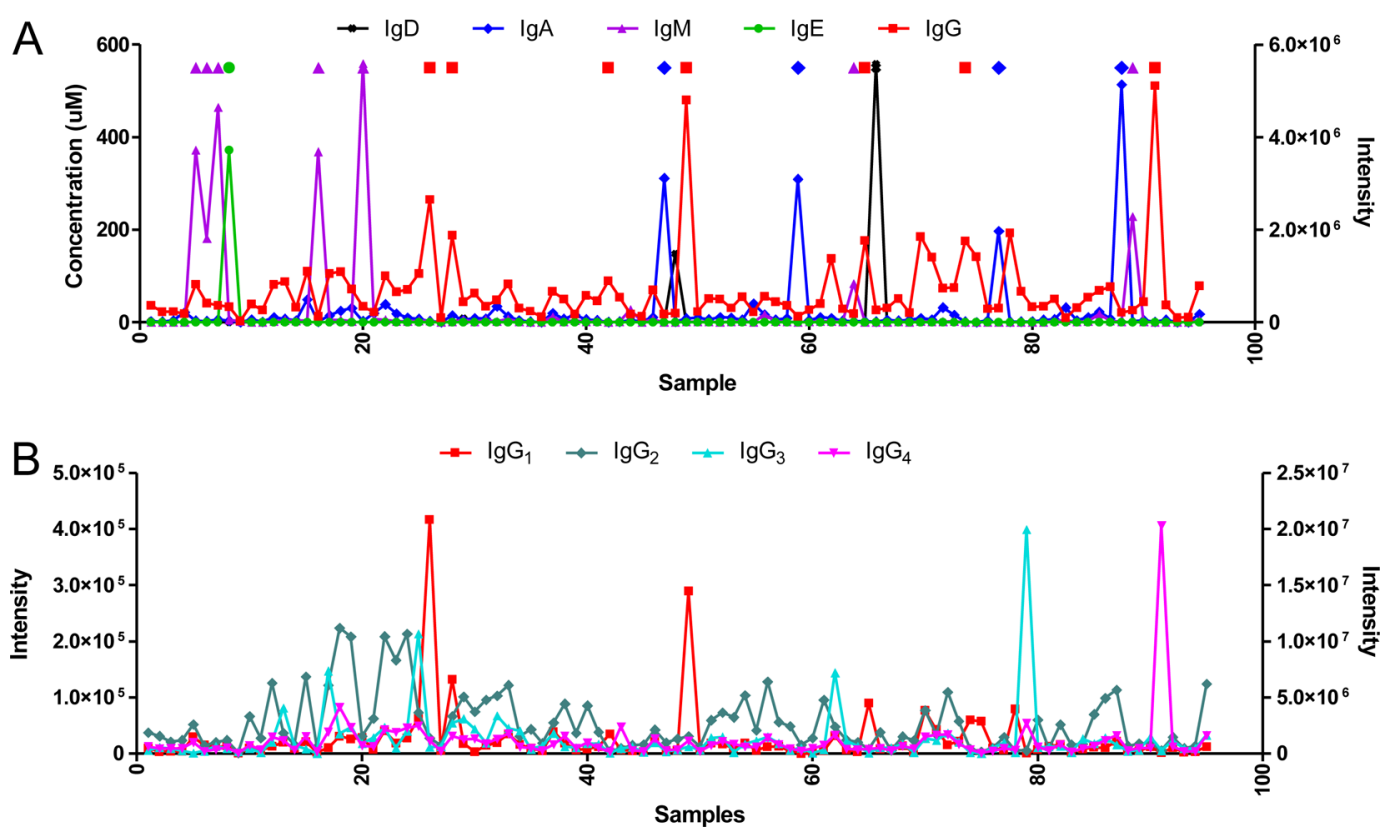

Figure 5. Data from undepleted sera for intact immunoglobulins. Abundances for (A) the main immunoglobulin classes $\operatorname{IgG}, \operatorname{IgA}, \operatorname{IgM}, \operatorname{IgE}$, and $\operatorname{IgD}$. Samples that were known to contain M-proteins in excess of $10 \mathrm{~g} / \mathrm{L}$ from available clinical data are highlighted with a marker matching for the class in the top of the figure. Data for IgD is plotted with uncalibrated intensity data on the right $Y$-axis. The other panel (B) shows data for subclasses IgG1, IgG2, IgG3, and IgG4. All data are uncalibrated intensity data, and those for IgG4 are on the right $Y$-axis.

not yet accounted for in the SRM transition set have been annotated in Table S-1 in the Supporting Information. Peptides with common polymorphisms, interfering peaks, or other inconsistencies were removed from the final computation method of sFLC. For incidental polymorphisms, concentrations can still be determined based on data from only the wild-type peptide. In our cohort, the polymorphisms that were found did not affect the light chain and computation of sFLC.

Patient Data. The pairwise comparison of the current SRM assay with the Freelite assay (Figure 4) and the N-latex assay (Figure S-4 in the Supporting Information) shows that all tests give comparable results for most samples. Data from the healthy controls are included in Figure 4 and Figure S-4 in the Supporting Information but were also summarized in Table S-2 in the Supporting Information. Only patients with a gammopathy involving one of the immunoglobulin classes that was not included in our depletion protocol had deviating results (affected points highlighted in red, intact immunoglobulins confirmed in depleted and whole serum data). Although the current method allows the identification of these samples as abnormal, depletion of the additional classes of immunoglobulin is required for proper quantification of these samples. This requires feasible adaptations in the sample preparation protocol.

We computed the $r^{2}$ for a linear fit of $\kappa, \lambda$, or $\kappa / \lambda$ ratio between log transformed data derived from the SRM and nephelometry assays, excluding the samples that could not be completely depleted. We obtained $r^{2}$ values ranging between 0.81 and 0.96 and slopes between 0.58 and 0.81 , as annotated in Figure 4 and Figure S-4 in the Supporting Information. Thus, the different methods do produce results that are correlated which each other. However, the slopes deviate from 1 , which indicate that the methods yield data that has an offset from each other between the methods. The data show that, for a given sample, results for both $\kappa$ and $\lambda$ chains rank as Freelite $>$ $\mathrm{N}$-Latex $>$ SRM. These differences complicate the comparison of data from different platforms, as mentioned in the introduction, and illustrate the need for harmonization. The cause of the differences is not known, but it may relate to the source, type, or other heterogeneities between the calibrants that are used on the different systems.

Serum protein electrophoresis (SPE) is an additional tool for sFLC quantification but due to limits in sensitivity only in samples with sufficiently high levels of sFLC. In Table S-3 in the Supporting Information, we list four such sera from the cohort, in which can be observed that the SRM data are in better agreement with the SPE result than either one of the nephelometric assays. Moreover, the nephelometry platforms also show considerable disagreement among each other, further bolstering confidence in the data from SRM and SPE.

As the SRM approach can also be well suited for the quantitation of intact immunoglobulins, we also processed whole serum from every patient to quantify intact IgA, IgG, $\mathrm{IgE}$, and IgM. Only qualitative measurements were included for $\mathrm{IgD}$ as well as subclass specific peptides for $\mathrm{IgG}_{1-4}$. As shown in Figure 5, samples with known M-proteins (>10 g/L) are readily identified by SRM and IgG M-proteins can be resolved to one of its subclasses in panel B. For the CRM serum, the data could be compared to the certified values accompanying the serum. As shown in Table S-4 in the Supporting Information, we found good agreement for IgA and IgM and a somewhat lower value for IgG.

Data Analysis. The richness of the data is a strength of the SRM approach but could also be perceived as a liability. Users should not routinely be exposed to the complexities of the underlying data set. It would be desirable to automate data analysis and quality control procedures and report a final result at the desired level of detail. This report could be associated with a quality metric to confirm that all cross-checks remained within bounds, with no suspicion of polymorphisms or other irregularities. In the current paper, data analysis was mostly performed manually in a spreadsheet and a robust platform for 
an automated approach remains to be established. Moreover, tolerance limits on all quality metrics remain to be thoroughly established based on routine operation of the assay.

Accuracy. The offset between results from the nephelometric assays, SRM and SPE illustrate that it is challenging to properly calibrate a platform for a heterogeneous analyte like sFLC. While the agreement between SPE and SRM shown in Table S-3 in the Supporting Information suggests that SRM is less sensitive to such heterogeneities than immunoassays, and remaining differences between, for example, cognate peptide pairs in SRM data show that also the accuracy in SRM could be improved. The comparison of accuracy and standardization of the techniques is hampered by the lack of an internationally accepted sFLC reference material. We believe the SRM approach has the potential to become a reference method that can harmonize data from different analytical platforms. However, we also believe that the SRM workflow needs more refinement and maturity before this goal can be achieved.

Outlook. The data shown in this paper illustrates the potential of an SRM method in the quantification of intact immunoglobulins and sFLC. The method delivers data comparable to the current standard nephelometric assays but without some of the drawbacks encountered in affinity-based methods. Moreover, as clinical laboratories introduce mass spectrometry instrumentation, it is increasingly attractive to transfer assays to this equipment. Our measurements illustrate that the assay is readily portable to instruments from different vendors.

For future work on this SRM assay for sFLC, we would recommend modifications that could improve to several aspects of the assay. Some peptides were rejected from the set used for quantification and could be omitted from the set of reference peptides, while standards should be added for $\operatorname{IgD}$ and $\operatorname{IgE}$. The peptides VPTGGVEEGLLER, TPECPSHTQPLGVYLLTPAVQDLWLR, and AVHEAASPSQTVQR performed well for this purpose.

Improvements may be achieved in cost and efficiency as well. Conservative conditions were chosen for LC runtime $(1 \mathrm{~h} /$ sample) and trypsin digestion (overnight). However, other studies as well as pilot data from our lab show that a shorter digestion of $3 \mathrm{~h}$ and reduction of the gradient length to $10 \mathrm{~min}$ can produce acceptable results.

In nephelometric assays, the performance relies on antibody reagents which may suffer from batch-to-batch variations, especially in the case of polyclonal antibodies. Moreover, assay antibodies may fail to interact as expected with their heterogeneous analytes or suffer from antigen excess problems when analyte concentrations are high. As the nephelometric assays yield a single numeric result, there is often no hint from the data that the measurement may have been anomalous.

The only affinity interaction remaining in the SRM workflow is the depletion of intact immunoglobulins. This step could be implemented with monoclonal antibodies, which would make the procedure more resistant against batch-to-batch variations. While problems in the depletion are visible in the data, a more complete depletion of all isotypes would result in a better accuracy of the results, especially in the case of gammopathies belonging to such isotypes. Other or additional depletion reagents may be helpful, but alternative approaches to the depletion of intact immunoglobulins may also be of interest, such as diafiltration of high molecular weight proteins from a serum sample or immunoprecipitation of sFLC with immobilized affinity reagents followed by SRM analysis of the bound material. The efficacy and cost-effectiveness of such alternative sample preparations remains to be shown, while affinity methods should also not inherit some of the drawbacks associated with the nephelometric immunoassays. Thus, while specific pitfalls remain in SRM assays, such as problems in sample preparation or polymorphisms, most issues can be revealed from the integrated data set.

\section{CONCLUSION}

The work in this paper shows that SRM is a promising method for the quantitation of immunoglobulins and sFLC. While additional optimizations would be needed, the data already show that sFLC measurements with SRM were comparable to those obtained by nephelometry. SRM has a wide measurement range and is not prone to analytical issues such as nonlinearity and antigen excess. Before the method can be implemented in a clinical context, additional validations on multiple instruments and sites are required. If such studies provide satisfactory results, we believe that our approach can be developed to either a reference method for the characterization of reference materials and challenging samples or a substitute for nephelometric tests for immunoglobulins and sFLC.

\section{ASSOCIATED CONTENT}

\section{S Supporting Information}

Quality control data, equations, additional comparisons between SRM and nephelometry results, and a table of peptides incorporated in the SRM method with associated data. The Supporting Information is available free of charge on the ACS Publications website at DOI: 10.1021/acs.analchem.5b01263.

\section{AUTHOR INFORMATION}

\section{Corresponding Author}

*Phone: +31-10-7038069. Fax: +31-10-7044365. E-mail: t. luider@erasmusmc.nl.

\section{Author Contributions}

${ }$ M.M.V. and J.F.M.J. contributed equally.

Notes

The authors declare no competing financial interest.

\section{ACKNOWLEDGMENTS}

This work was financially supported by stipends from the E.C. Noyons Foundation and the Dutch Foundation for Quality Assessment in Medical Laboratories (SKML), section Humoral Immunity.

\section{REFERENCES}

(1) Dimopoulos, M.; Kyle, R.; Fermand, J. P.; Rajkumar, S. V.; San Miguel, J.; Chanan-Khan, A.; Ludwig, H.; Joshua, D.; Mehta, J.; Gertz, M.; Avet-Loiseau, H.; Beksac, M.; Anderson, K. C.; Moreau, P.; Singhal, S.; Goldschmidt, H.; Boccadoro, M.; Kumar, S.; Giralt, S.; Munshi, N. C.; Jagannath, S. Blood 2011, 117, 4701-4705.

(2) Rajkumar, S. V.; Dimopoulos, M. A.; Palumbo, A.; Blade, J.; Merlini, G.; Mateos, M. V.; Kumar, S.; Hillengass, J.; Kastritis, E.; Richardson, P.; Landgren, O.; Paiva, B.; Dispenzieri, A.; Weiss, B.; LeLeu, X.; Zweegman, S.; Lonial, S.; Rosinol, L.; Zamagni, E.; Jagannath, S.; Sezer, O.; Kristinsson, S. Y.; Caers, J.; Usmani, S. Z.; Lahuerta, J. J.; Johnsen, H. E.; Beksac, M.; Cavo, M.; Goldschmidt, H.; Terpos, E.; Kyle, R. A.; Anderson, K. C.; Durie, B. G.; Miguel, J. F. Lancet Oncol. 2014, 15, e538-548.

(3) Jelinek, A. G.; Bachmann, L. M. Clin. Chem. 2014, 60, 13751378. 
(4) Tate, J.; Mollee, P.; Johnson, R. Clin. Biochem. Rev. 2009, 30, 8991.

(5) Jacobs, J. F. M.; Hoedemakers, R. M. J.; Teunissen, E.; van der Molen, R. G.; te Velthuis, H. Clin. Chim. Acta 2012, 413, 1708.

(6) de Kat Angelino, C. M.; Raymakers, R.; Teunesen, M. A.; Jacobs, J. F.; Klasen, I. S. Clin. Chem. 2010, 56, 1188-1190.

(7) Maisnar, V.; Tichy, M.; Stulik, J.; Vavrova, J.; Friedecky, B.; Palicka, V.; Spirkova, J.; Zaloudkova, L.; Hernychova, L.; Spacilova, J.; Buchler, T.; Hajek, R. Clin. Biochem. 2011, 44, 403-405.

(8) Murray, D. L.; Ryu, E.; Snyder, M. R.; Katzmann, J. A. Clin. Chem. 2009, 55, 1523-1529.

(9) Graziani, M. S.; Merlini, G. Expert Rev. Mol. Diagn. 2014, 14, 5566.

(10) Hutchison, C. A.; Harding, S.; Hewins, P.; Mead, G. P.; Townsend, J.; Bradwell, A. R.; Cockwell, P. Clin. J. Am. Soc. Nephrol. 2008, 3, 1684-1690.

(11) Bradwell, A. R.; Carr-Smith, H. D.; Mead, G. P.; Tang, L. X.; Showell, P. J.; Drayson, M. T.; Drew, R. Clin. Chem. 2001, 47, 673680.

(12) Dispenzieri, A.; Kyle, R.; Merlini, G.; Miguel, J. S.; Ludwig, H.; Hajek, R.; Palumbo, A.; Jagannath, S.; Blade, J.; Lonial, S.; Dimopoulos, M.; Comenzo, R.; Einsele, H.; Barlogie, B.; Anderson, K.; Gertz, M.; Harousseau, J. L.; Attal, M.; Tosi, P.; Sonneveld, P.; Boccadoro, M.; Morgan, G.; Richardson, P.; Sezer, O.; Mateos, M. V.; Cavo, M.; Joshua, D.; Turesson, I.; Chen, W.; Shimizu, K.; Powles, R.; Rajkumar, S. V.; Durie, B. G. Leukemia 2009, 23, 215-224.

(13) Durie, B. G.; Harousseau, J. L.; Miguel, J. S.; Blade, J.; Barlogie, B.; Anderson, K.; Gertz, M.; Dimopoulos, M.; Westin, J.; Sonneveld, P.; Ludwig, H.; Gahrton, G.; Beksac, M.; Crowley, J.; Belch, A.; Boccadaro, M.; Cavo, M.; Turesson, I.; Joshua, D.; Vesole, D.; Kyle, R.; Alexanian, R.; Tricot, G.; Attal, M.; Merlini, G.; Powles, R.; Richardson, P.; Shimizu, K.; Tosi, P.; Morgan, G.; Rajkumar, S. V. Leukemia 2006, 20, 1467-1473.

(14) Abraham, R. S.; Charlesworth, M. C.; Owen, B. A.; Benson, L. M.; Katzmann, J. A.; Reeder, C. B.; Kyle, R. A. Clin. Chem. 2002, 48, $1805-1811$.

(15) Bosmann, M.; Kossler, J.; Stolz, H.; Walter, U.; Knop, S.; Steigerwald, U. Clin. Chem. Lab. Med. 2010, 48, 1419-1422.

(16) Jacobs, J. F.; Hoedemakers, R. M.; Teunissen, E.; te Velthuis, H. Clin. Chem. Lab. Med. 2014, 52, 1-7.

(17) Tate, J. R.; Mollee, P.; Dimeski, G.; Carter, A. C.; Gill, D. Clin. Chim. Acta 2007, 376, 30-36.

(18) Vercammen, M.; Meirlaen, P.; Broodtaerts, L.; Broek, I. V.; Bossuyt, X. Clin. Chim. Acta 2011, 412, 1798-1804.

(19) te Velthuis, H.; Knop, I.; Stam, P.; van den Broek, M.; Bos, H. K.; Hol, S.; Teunissen, E.; Fischedick, K. S.; Althaus, H.; Schmidt, B.; Wagner, C.; Melsert, R. Clin. Chem. Lab. Med. 2011, 49, 1323-1332. (20) Hoedemakers, R. M.; Pruijt, J. F.; Hol, S.; Teunissen, E.; Martens, H.; Stam, P.; Melsert, R.; te Velthuis, V. H. Clin. Chem. Lab. Med. 2012, 50, 489.

(21) Pretorius, C. J.; Klingberg, S.; Tate, J.; Wilgen, U.; Ungerer, J. P. Ann. Clin. Biochem. 2012, 49, 450-455.

(22) Lock, R. J.; Saleem, R.; Roberts, E. G.; Wallage, M. J.; Pesce, T. J.; Rowbottom, A.; Cooper, S. J.; McEvoy, E. D.; Taylor, J. L.; Basu, S. Ann. Clin. Biochem. 2013, 50, 255-261.

(23) Lavatelli, F.; Brambilla, F.; Valentini, V.; Rognoni, P.; Casarini, S.; Di Silvestre, D.; Perfetti, V.; Palladini, G.; Sarais, G.; Mauri, P.; Merlini, G. Biochim. Biophys. Acta, Proteins Proteomics 2011, 1814, 409-419.

(24) Ladwig, P. M.; Barnidge, D. R.; Snyder, M. R.; Katzmann, J. A.; Murray, D. L. Clin. Chem. 2014, 60, 1080-1088.

(25) Remily-Wood, E. R.; Benson, K.; Baz, R. C.; Chen, Y. A.; Hussein, M.; Hartley-Brown, M. A.; Sprung, R. W.; Perez, B.; Liu, R. Z.; Yoder, S. J.; Teer, J. K.; Eschrich, S. A.; Koomen, J. M. Proteomics: Clin. Appl. 2014, 8, 783-795.

(26) Barnidge, D. R.; Tschumper, R. C.; Theis, J. D.; Snyder, M. R.; Jelinek, D. F.; Katzmann, J. A.; Dispenzieri, A.; Murray, D. L. J. Proteome Res. 2014, 13, 1905-1910.

(27) Picotti, P.; Aebersold, R. Nat. Methods 2012, 9, 555-566.
(28) Gerber, S. A.; Rush, J.; Stemman, O.; Kirschner, M. W.; Gygi, S. P. Proc. Natl. Acad. Sci. U. S. A. 2003, 100, 6940-6945.

(29) Zegers, I.; Keller, T.; Schreiber, W.; Sheldon, J.; Albertini, R.; Blirup-Jensen, S.; Johnson, M.; Trapmann, S.; Emons, H.; Merlini, G.; Schimmel, H. Clin. Chem. 2010, 56, 1880-1888.

(30) Katzmann, J. A.; Clark, R. J.; Abraham, R. S.; Bryant, S.; Lymp, J. F.; Bradwell, A. R.; Kyle, R. A. Clin. Chem. 2002, 48, 1437-1444.

(31) MacLean, B.; Tomazela, D. M.; Shulman, N.; Chambers, M.; Finney, G. L.; Frewen, B.; Kern, R.; Tabb, D. L.; Liebler, D. C.; MacCoss, M. J. Bioinformatics 2010, 26, 966-968.

(32) Wahner-Roedler, D. L.; Kyle, R. A. Best practice \& research. Clinical haematology 2005, 18, 729-746.

(33) Lefranc, M. P.; Giudicelli, V.; Ginestoux, C.; Jabado-Michaloud, J.; Folch, G.; Bellahcene, F.; Wu, Y.; Gemrot, E.; Brochet, X.; Lane, J.; Regnier, L.; Ehrenmann, F.; Lefranc, G.; Duroux, P. Nucleic Acids Res. 2009, 37, D1006-1012.

(34) Kent, W. J. Genome Res. 2002, 12, 656-664.

(35) Kent, W. J.; Sugnet, C. W.; Furey, T. S.; Roskin, K. M.; Pringle, T. H.; Zahler, A. M.; Haussler, D. Genome Res. 2002, 12, 996-1006.

(36) Johnson, G.; Wu, T. T. Nucleic Acids Res. 2000, 28, 214-218.

(37) Exome Aggregation Consortium. Cambridge, MA, 2015. http:// exac.broadinstitute.org/. 